\title{
Electron Scattering from Neon Via Effective Range Theory
}

\author{
Kamil Fedus
}

Received: 20 August 2014 / Published online: 9 October 2014

(C) The Author(s) 2014. This article is published with open access at Springerlink.com

\begin{abstract}
Elastic cross-sections for electron scattering on neon from 0 energy up to $16 \mathrm{eV}$ are analyzed by an analytical approach to the modified effective range theory (MERT). It is shown that energy and angular variations of elastic differential, integral and momentum transfer crosssections can be accurately parameterized by six MERT coefficients up to the energy threshold for the first Feshbach resonance. MERT parameters are determined empirically by numerical comparison with large collection of available experimental data of elastic total (integral) cross-sections. The present analysis is validated against numerous electron beams and swarm experiments. The comparison of derived MERT parameters with those found for other noble gases, helium, argon and krypton, is done. The derived scattering length (for the $s$-partial wave) in neon, $0.227 a_{0}$, agrees well with recent theories; it is small but, differently from Ar and $\mathrm{Kr}$, still positive. Analogue parameters for the $p$-wave and the $d$-wave are negative and positive respectively for all the four gases compared.
\end{abstract}

Keywords Electron elastic scattering $\cdot$ Neon · Modified effective range theory

\section{Introduction}

Neon is used as an admixture in numerous gas-discharge applications; it is also an important gas in the field of experimental and theoretical electron-scattering studies. Already

\footnotetext{
K. Fedus ( $\varangle$ )

Institute of Physics, Faculty of Physics, Astronomy and

Informatics, Nicolaus Copernicus University, Grudziadzka 5,

87-100 Torun, Poland

e-mail: kamil@ fizyka.umk.pl
}

some 20 years ago, it has been proposed [1] as a secondary standard, together with helium, for normalizing relative measurements. However, despite a great attention devoted to this atom over the years, the scattering in very low-energy range is still a challenging task both experimentally and theoretically. Therefore, the interaction of slow electrons with Ne remains an active subject of research in particular, in the light of the quest for establishing a complete and consistent electron cross-sectional data sets for low-temperature plasma modelling [2].

Recently, Shigemura et al. [3] employing threshold photo electron source were able to measure $\mathrm{Ne}$ total cross-sections (TCS) down to $7 \mathrm{meV}$. Linert et al. [4] and Cho et al. [5] using magnetic angle-changer were able to extend differential cross-section (DCS) measurements to $180^{\circ}$ scattering angle that was inaccessible in the past. New experimental results remain consistent with older measurements of elastic TCS [6-13] and DCS $[1,11,14,15]$. On the other hand, the recent experimental achievements, especially at high angles, became a stringent test for theories. It is well-known that at large distances $r$ from the target, the interaction of neutral atom with slow electrons is governed by the adiabatic polarization potential $V_{\mathrm{pol}}=-\alpha / 2 r^{4}$ where $\alpha$ is the dipole polarizability. However, at shorter distances, the exact form of the potential, strictly speaking, is not defined since the complex many body interactions play a dominant role.

Different strategies have been designed to describe the short-range correlation effects between incoming slow electron and atomic charge cloud. The most recent theoretical approaches are based on Dirac equation. These include works of Cheng et al. [16] using box-variational method, Zatsarinny and Bartschat $[17,18]$ using $B$-spline $R$-matrix method and relativistic model of McEachran et al. [5] . Older approaches focused on describing low-energy elastic scattering include ab initio calculations of Saha [19, 20]; 
polarized orbital calculations of McEachran and Stauffer [21-23], Dasgupta and Bahtia [24], Garbaty and LaBahn [25] and Thomson [26]; and $R$-matrix calculations of Fon and Berrington [27]. These theories differ both in the asymptotic forms on the polarization potential and on details of the short-range interaction. Moreover, the inclusion of short-range effects for many-electron atoms in the frame of many-body theories is not simple and it is computationally expensive. Therefore, a great value in understanding angular and energy variations of low-energy cross-sectional data can be brought by semi-empirical analysis of experimental results. Such approaches were undertaken in the case of $\mathrm{e}^{-}-\mathrm{Ne}$ collisions by Bottcher [28] using model potential fit, McDowell [29] and Williams [30] using phaseshift analysis, and O'Malley and Crompton [31] using their well-known modified effective range theory (MERT) [32, 33]. Particularly, in the latter work, the authors proposed a fully empirical version of MERT to describe the whole elastic range of $\mathrm{Ne}$ cross-sections using seven parameters (see appendix of ref. [31]). Unfortunately, this (ambitious) project remained somewhat unfinished.

Recently, in a couple of papers $[34,35]$, we showed that an alternative form of MERT proposed by Idziaszek and Karwasz [36, 37] describes very well the electron-elastic scattering from helium, argon and krypton almost up to the energy threshold for the first inelastic process. MERT is a simple non-relativistic model describing the scattering of spineless particles by a central long-range polarization potential $\left(V_{\text {pol }}\right)$ while the complex short-range effects are included within the frame of some boundary conditions imposed on the wave function. MERT provides different expressions for partial waves scattering phase shift as a function of wave number of electron $k$. However, in the derivation of original MERT expressions by O'Malley et al. [32], both the long-range and the short-range parts of the interaction were approximated by the effective range expansions. On the contrary, in the approach proposed by Idziaszek and Karwasz [36], the contribution of the longrange polarization interaction to the scattering phase shifts is solved exactly while the effective range approximation is introduced exclusively for the short-range part of the potential. This simple alteration with respect to the original model allowed extending the applicability of semi-empirical MERT analysis to much higher energies than it has been considered so far (e.g. much below $1 \mathrm{eV}$ in noble gases; see ref. [38]). Moreover, we showed that this approach could be successfully used to describe electron-elastic scattering from quasi-spherical (with $\Sigma$ symmetry) molecules like $\mathrm{H}_{2}$ and $\mathrm{CH}_{4}[34,39]$ in relatively wide energy range. Consequently, we demonstrated that using only one equation with few adjustable parameters to describe partial waves scattering phase shifts, it is possible to parameterize almost the entire set of elastic differential, integral and momentum transfer cross-sections for electrons collisions with light spherical targets characterized by low dipole polarizability.

This paper aims to complete our effective range analysis on noble gases ( $\mathrm{He}$ and $\mathrm{Ar}$ in [34] and $\mathrm{Kr}$ in [35]) by studying $\mathrm{e}^{-}-\mathrm{Ne}$ elastic collisions. Using the most recent experimental results, it is shown that just as for the other noble gases studied by us in the past, only six parameters of the effective range expansions are needed to describe both energy and angular dependences of scattering cross-sections for $\mathrm{e}^{-}-\mathrm{Ne}$ interaction up to the threshold for the first Feshbach resonance $(<16 \mathrm{eV})$. In addition, a comparison of derived parameters of the effective range expansions for all studied atoms, $\mathrm{He}, \mathrm{Ne}, \mathrm{Ar}$ and $\mathrm{Kr}$, is done.

The paper is organized as follows: In Section 2, the principles of modified effective range theory are briefly described in the context of electron-neon interaction. In Section 3, the effective range analysis of integral, momentum transfer and differential cross-sections is presented. The paper is accomplished with conclusions in Section 4.

\section{Theoretical Model}

Details of the analytical approach to MERT have been described in previous papers $[34,36]$. In this section, only a very brief account will be recalled. Current approach is based on properties of Mathieu functions-the analytical solutions of the radial Schrödinger equation with pure polarization potential $\left(V_{\mathrm{pol}}=\alpha / 2 r^{4}\right)$. The scattering phase shift $\eta_{l}$ experienced by $l$ th partial wave is given by the following relation (in atomic units):

$\tan \eta_{l}(k)=\frac{m_{l}^{2}-\tan ^{2} \delta_{l}+\widetilde{B}_{l} \tan \delta_{l}\left(m_{l}^{2}-1\right)}{\tan \delta_{l}\left(1-m_{l}^{2}\right)+\widetilde{B}_{l}\left(1-m_{l}^{2} \tan ^{2} \delta_{l}\right)}$,

where $\delta_{l}=\pi\left(v_{l}-l-1 / 2\right) / 2$. Here, $m_{l}(k)$ and $v_{l}(k)$ (characteristic exponent of Mathieu functions) denote the energy-dependent parameters which have to be determined numerically from analytical properties of Mathieu functions (see ref. $[34,36]$ for details of numerical procedure). The contribution of the short-range interaction is given by parameter $\widetilde{B}_{l}(k)$. The latter is approximated by the quadratic effective range expansion:

$\widetilde{B}_{l}(k)=B_{l}(0)+R_{l} R^{*} k^{2} / 2+\ldots$,

where $B_{l}(0)$ is the zero-energy contribution and $R_{l}$ can be interpreted as the effective range for a given partial wave. In the particular case of $l=0, B_{0}$ can be expressed in terms of $A_{0}$, the s-wave scattering length, as: $B_{0}=-R^{*} / A_{0}$, where $R^{*}=\sqrt{\alpha}$. In the calculations, an experimental value of the dipole polarizability of neon, $\alpha=2.571 a_{0}^{3}$ [40], is used. The parameters of the effective range expansions, $B_{l}$ and 
$R_{l}$, have to be determined empirically by comparison with some experimental data.

Integral elastic $\left(\sigma_{\mathrm{IE}}\right)$, momentum transfer $\left(\sigma_{\mathrm{MT}}\right)$ and differential elastic $(d \sigma / d \omega)$ cross-sections are calculated using the standard partial wave expansions:

$\sigma_{\mathrm{IE}}=\frac{4 \pi}{k^{2}} \sum_{l=0}^{\infty}(2 l+1) \sin \eta_{l}(k)$,

$\sigma_{\mathrm{MT}}=\frac{4 \pi}{k^{2}} \sum_{l=0}^{\infty}(l+1) \sin \left[\eta_{l}(k)-\eta_{l+1}(k)\right]$,

$d \sigma / d \omega=\frac{1}{k^{2}}\left|\sum_{l=0}^{\infty}(2 l+1) \exp \left(i \eta_{l}\right) \sin \left(\eta_{l}\right) P_{l}(\cos \theta)\right|^{2}$

where $\theta$ is the scattering angle and $P_{l}(\cos \theta)$ are the Legendre polynomials.

This semi-empirical model has been applied to verify that in the regime of energies for elastic scattering $(<16 \mathrm{eV})$, the leading contributions for neon come from $s, p$ and $d$ waves $(l=0,1,2)$ while the contribution of higher partial waves is small and can be described by taking only the leading order contribution to the phase shift:

$\tan \eta_{l}(k)=\frac{\pi \alpha k^{2}}{8(l-1 / 2)(l+1 / 2)(l+3 / 2)}$, for $l>2$.

Equation (6) is exact at the low-energy limit [32], and it can be also reproduced using a first-order Born approximation. Alternatively to Eq. (6), one can also describe the contribution of higher partial waves $(l>2)$ taking into account the higher-order energy terms in the long-range forces contribution to the phase shifts given by Ali and Fraser, i.e. Eqs. 12-14 in ref. [41]. However, I checked that the inclusion of these higher order terms is negligible in considered energy range and simple Eq. (6) is sufficient to include the contribution of higher partial waves $(l>2)$ which cannot overcome the repulsion of the centrifugal barrier and probe the region of the short-range interactions. Moreover, I also checked that the contribution of quadrupole polarizability terms present in Ali and Fraser's expressions [41] are negligible for neon in considered energy range.

\section{MERT Analysis}

The scattering-phase shifts can be derived by fitting MERT either to the experimental elastic TCS (as in ref. [34] for $\mathrm{He}, \mathrm{Ar}$ ), to DCS (as in ref. [35] for $\mathrm{Kr}$ ) or to momentum transfer cross-sections (MTCS). In this work, the fact is used that experimental low-energy TCS for Ne are generally in very good agreement with each other. Moreover, TCS are measured in absolute way, and in low-energy range, they correspond to elastic integral cross-sections. Therefore, the scattering phase shifts were determined by performing simultaneous robust fit of Eq. (4) to large collection of TCS data sets (using MATLAB routine for nonlinear least-square regression of multiple data sets). No additional constraints on fitting parameters were imposed. Figure 1a presents the results of such fit for data below the first Fesbach resonance $(<16 \mathrm{eV})$. The obtained curve stays within $10 \%$ agreement with all experimental points, i.e. within the combined error bar of separate measurements. In Fig. 1b, the contribution of $s$-, $p$ - and $d$-waves to TCS is also shown.

The $s$-wave scattering is dominant in whole energy range considered and the contribution of higher partial waves can be practically neglected up to $2 \mathrm{eV}$ of impact energy. The latter result stays in perfect agreement with calculations of O'Malley and Crompton [31] using original MERT approach, but applied only up to $2 \mathrm{eV}$. The present results confirm also a characteristic behaviour of $p$-wave contribution, which reaches 0 at around $1.2 \mathrm{eV}$. However, this

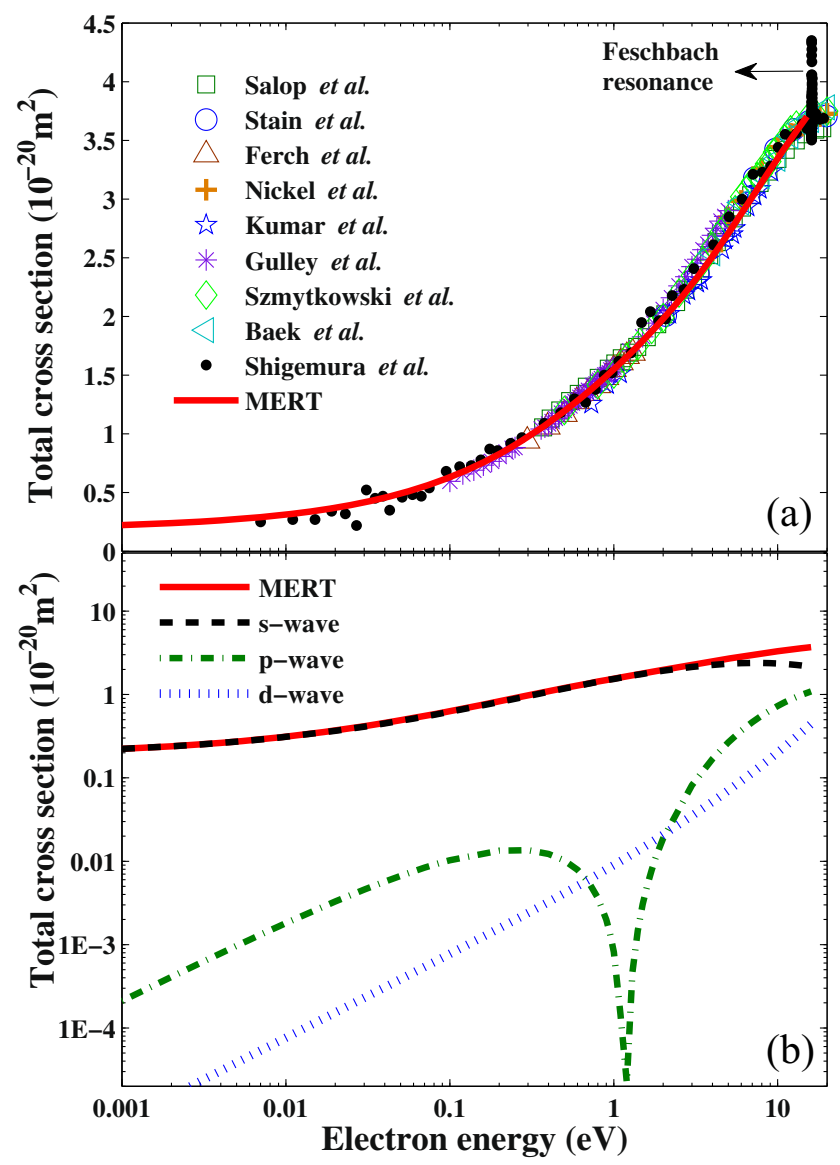

Fig. 1 a MERT simultaneous robust fit (solid line) to experimental total cross-sections (TCS) for $e^{-}-\mathrm{Ne}$ collisions. The experimental data are from Salop and Nakano [6], Stein et al. [7] , Ferch and Raith [8] , Nickel et al. [9], Kumar et al. [10] , Gulley et al. [11], Szmytkowski et al. [12] , Baek and Grosswendt [13] , and Shigemura et al. [14] b MERT-derived contribution of $s-, p$ - and $d$ - partial waves to TCS 
Table 1 Parameters of the effective range expansions for electron scattering from noble gases: $A_{0}=-R^{*} / B_{0}$ (the scattering length), $B_{1}$ (zero energy contribution for $p$-wave), $B_{2}$ (zero energy contribution for $d$-wave), $R_{0}$ ( $s$-wave effective range), $R_{1}$ ( $p$-wave effective range) and $R_{2}$ ( $d$-wave effective range)

\begin{tabular}{lllllll}
\hline & $A_{0}\left(a_{0}\right)$ & $B_{1}$ & $B_{2}$ & $R_{0}\left(a_{0}\right)$ & $R_{1}\left(a_{0}\right)$ & - \\
\hline$e^{-}-\mathrm{He}[34]$ & 1.186 & -20 & - & 0.01 & -100 \\
$e^{-}-\mathrm{Ne}$ & 0.227 & -0.231 & 0.001 & 3.697 & -0.028 & 0.361 \\
$e^{-}-\operatorname{Ar}[34]$ & -1.51 & -0.44 & 0.21 & -0.38 & 0.06 & 0.30 \\
$e^{-}-\operatorname{Kr}[35]$ & -3.486 & -0.599 & 0.125 & 0.533 & 0.039 \\
\hline
\end{tabular}

minimum is not visible in TCS due to the small contribution of the $p$-wave when compared to the $s$-wave at this energy range. We note, however, that in argon discharges, the 0 cross-section in the $p$-wave scattering reflects in a double electron temperature distribution [42]. Recent measurements in RF-driven discharges in neon [43] revealed an average temperature of $8 \mathrm{eV}$, but in a specific region of the discharge tube, it drops down to below $2 \mathrm{eV}$.

The derived six parameters of the effective range expansion are given in Table 1. In particular, the $s$-wave scattering length, $A_{0}=0.227 a_{0}$, is in very good agreement with previous experimental and theoretical determinations, giving the results between 0.2 and $0.3 a_{0}$. The present result is particularly close to $0.224 a_{0}$ given by the most recent calculations of Cheng et al. [16] who has designed the box variational method specifically to describe very low-energy $e^{-}-\mathrm{Ne}$ scattering. The extensive comparison of scattering lengths for neon obtained using different methods has been done in the latter work; therefore, it will not be repeated here.

Table 1 gives also parameters of the effective range expansions for other noble gases published in our previous articles [34, 35]. Using these factors, one can calculate the elastic cross-sections up to the energy threshold of the first inelastic process. However, at present, it is difficult to find any systematics in the calculated parameters-they reproduce the contribution of overall scattering potential, consisting of the static, exchange and polarization parts. The scattering length $A_{0}$ lowers systematically from about $1.2 a_{0}$ in $\mathrm{He}$ to $0.227 a_{0}$ in $\mathrm{Ne}$ to $-3.5 a_{0}$ in $\mathrm{Kr}$; several authors, see for example [44], related this tendency to rising polarizabities in the series $\mathrm{He}-\mathrm{Xe}$. An analogue parameter $B_{1}$ for the $p$-wave is negative while $B_{2}$ for the $d$-wave is positive for all the four gases compared. The other parameters from
Fig. 2 Comparison of $s-, p$ and $d$-wave scattering phase-shifts $\left(\eta_{0}, \eta_{1}\right.$ and $\left.\eta_{2}\right)$ derived in present MERT analysis with calculations of Garbaty and LaBahn [25], Fon and Berrington [27], McEachran and Stauffer [22] Dasgupta and Bhatia [24], Saha [19] and Cheng et al. [16]. The results of semi-empirical analysis of O'Malley and Crompton [31], Williams [30], Brewer et al. [15], Register and Trajmar [14] and Gulley et al. [11] are also presented. The $f$-wave phase shift $\left(\eta_{3}\right)$ calculated in the Born approximation using (6) is shown as well (note a different abscissa scale in the $f$-wave graph)

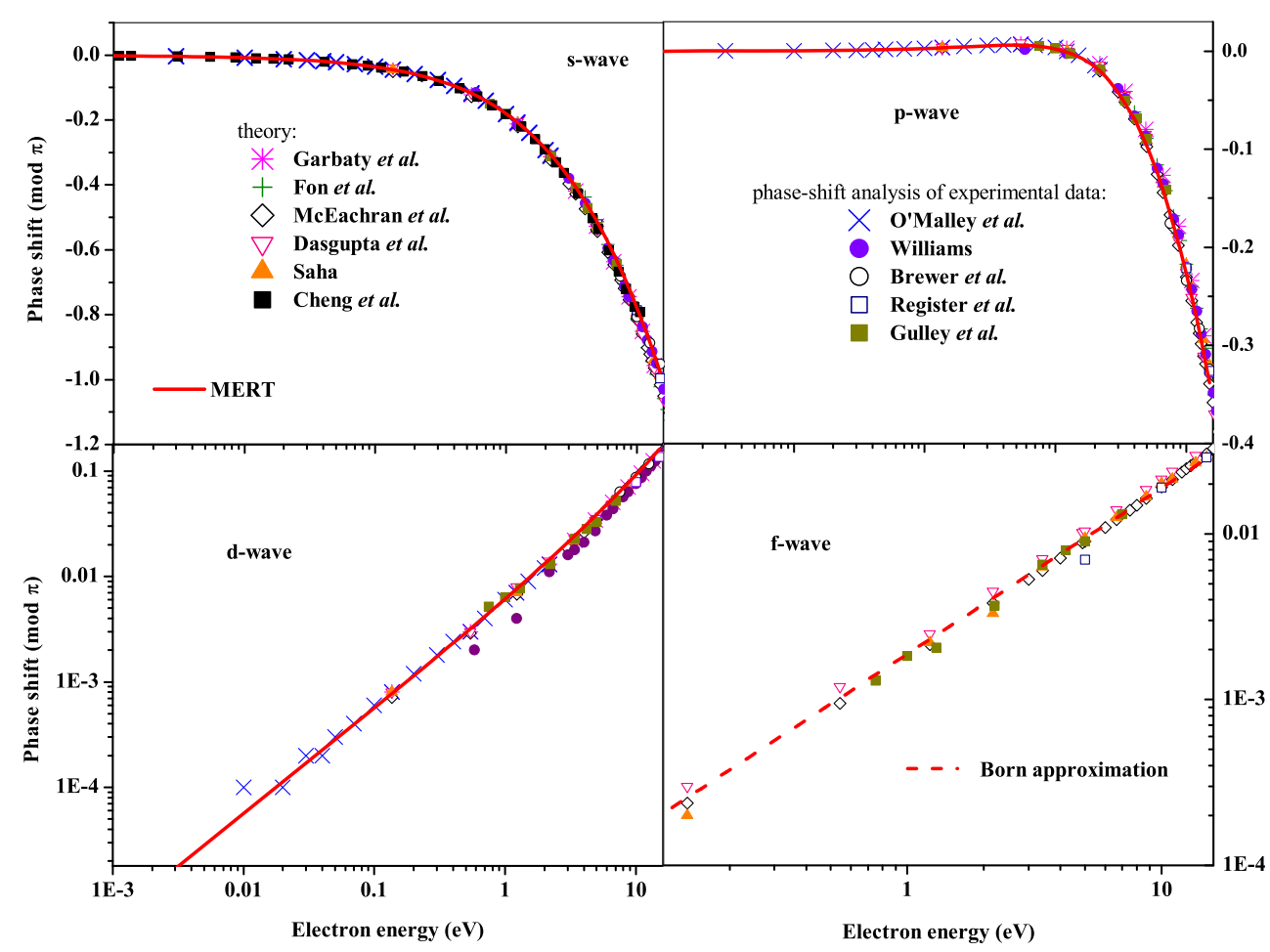


Table 1 does not show such regular behaviour. Nevertheless, the physical meaning of the $k^{2}$ terms in the expansion (2), representing the effective range corrections, is still to be understood. Moreover, the multi-term fit is always characterized by a certain portion of ambiguity since the large number of independent parameters can prevent their unique determination from experimental data. It is particularly important for helium where it is difficult to unambiguously determine both the $s$ - and the $p$-wave phase shifts due to the lack of a strong energy dependence of the cross-section [45]. Furthermore, the effective range corrections are relatively small in the low-energy regime in comparison to the leading contribution due to the $s$-wave scattering length and the $p$-/ $d$-wave zero-energy contributions. Consequently, $R_{l}$ terms can be strongly affected by the fitting procedure (e.g. a choice of data range for fit) and the measurement uncertainties in the experimental data. Therefore, the data shown in Table 1 can be still changed if new and more accurate experimental results will be provided in lower energy range.

The parameters from Table 1 for Ne were used in Eq. (1) to calculate the scattering phase shifts, and the results are compared with other theories in Fig. 2. It is clear that the agreement between the present calculations and the results reported by other authors is quite good, $5 \%$ consistency up to $10 \mathrm{eV}$ for $s$ - and $p$-waves. Our non-relativistic approach should be sufficient to describe scattering data since the spin-orbit splitting of the phase shifts is generally small in considered energy range [16]. Figure 2 compares also the $f$-wave phase shift calculated in the Born approximation using Eq. (6) with the chosen reference data. The perfect agreement confirms that the contribution of higher partial waves $(l>2)$, which are not distorted by the shortrange interaction, can be very well described using simple Eq. (6).

To validate additionally the present approach, the phase shifts shown in Fig. 2 were used to calculate elastic momentum transfer cross-sections using Eq. (4) and differential cross-sections using Eq. (5) to compare against numerous experimental and theoretical data. The results are shown in Figs. 3 and 4 respectively. Presently, calculated MTCS stays within $10 \%$ agreement with all swarm [46-51] and beam-derived $[4,5,11,14,15]$ experimental results below $10 \mathrm{eV}$ and within $15 \%$ for higher energies. Unlike the TCS, the MTCS is characterized by a small concave bend of the curve around $2-6 \mathrm{eV}$. MERT calculations indicate that the increasing $p$-wave contribution becomes comparable to decreasing $s$-wave in this energy range. When compared to theoretical results $[16,18,19,22,27]$, MERT is the most consistent with the recent model of Cheng et al. [16] up to $9 \mathrm{eV}$ of impact energy. However, for higher energies, their calculations start to decrease with energy (not shown here) what is inconsistent with the observation, and the present

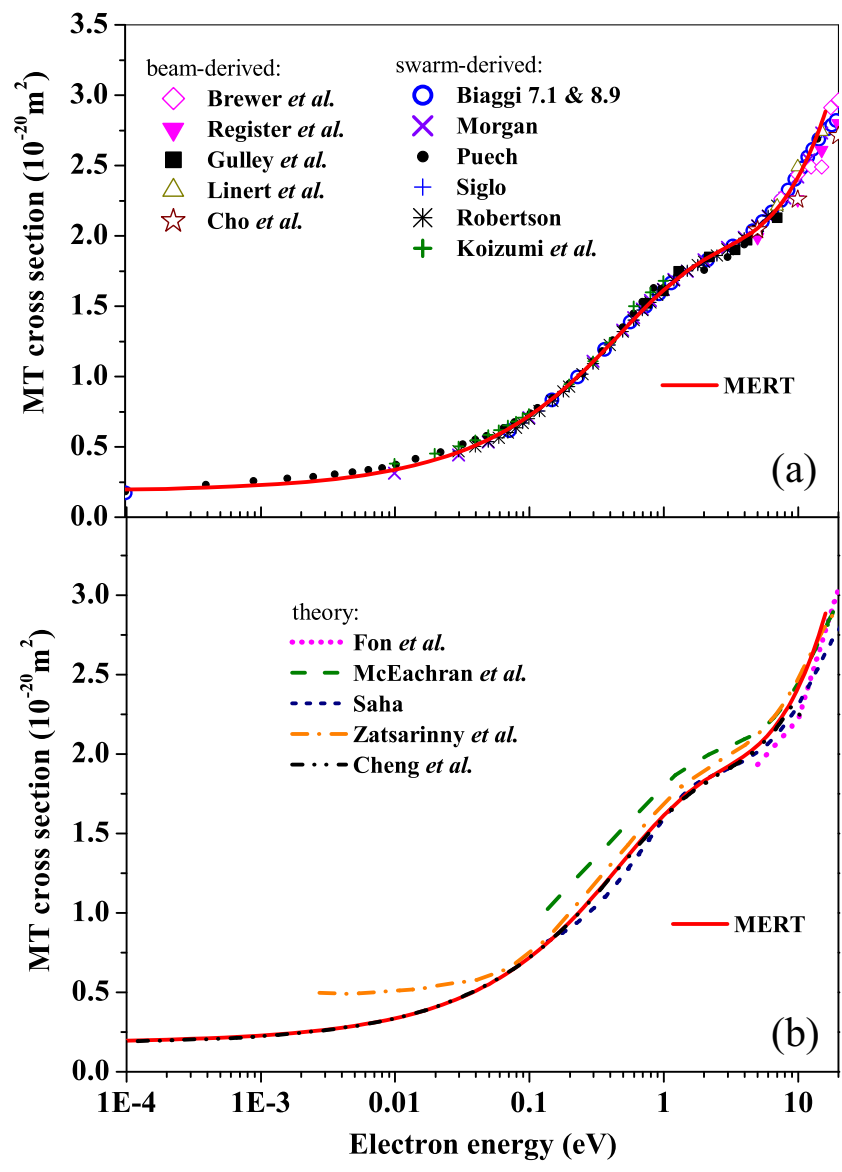

Fig. 3 a Experimental and $\mathbf{b}$ theoretical momentum transfer crosssections for elastic $e^{-}-\mathrm{Ne}$ scattering below $16 \mathrm{eV}$ compared with the present MERT calculations. The swarm-derived data are from Robertson [46], Kuizumi et al. [47], Biaggi [48], Morgan [49], Puech [50] and Siglo [51]. The beam-derived data are from Linert et al. [4], Cho et al. [5], Gulley et al. [11], Register and Trajmar [14] and Brewer et al. [15]. The theoretical data are from Cheng et al. [16], Zatsarinny and Bartschat [18], Saha [19], McEachran and Stauffer [22] and Fon and Berrington [27]

MERT becomes more comparable with the recent $B$-spline $R$-matrix, which are results of the study of Zatsarinny and Bartschat [18].

MERT-derived angular distributions of DCS shown in Fig. 4 are in very good agreement with all available experimental data $[1,4,5,11,14]$. These results show that our simple model using only six parameters is able to take into account not only energy but also the angular dependence of the scattering cross-sections. In particular, the present calculations are consistent with very low-energy data of Shi and Burrow [1]. To the best of our knowledge, their sets are so far the only experimental DCS available below $1 \mathrm{eV}$. Note also a very good agreement with high angle experimental data of Linert et al. [4] and Cho et al. [5]. 
Fig. 4 Differential

cross-section for elastic $e^{-}-\mathrm{Ne}$ scattering at electron energies between 0.25 and $15 \mathrm{eV}$ as a function of the scattering angle. The experimental data of Shi and Burrow [1], Linert et al. [4], Cho et al. [5], Gulley et al. [11] and Register and Trajmar [14] are compared with the present MERT calculations

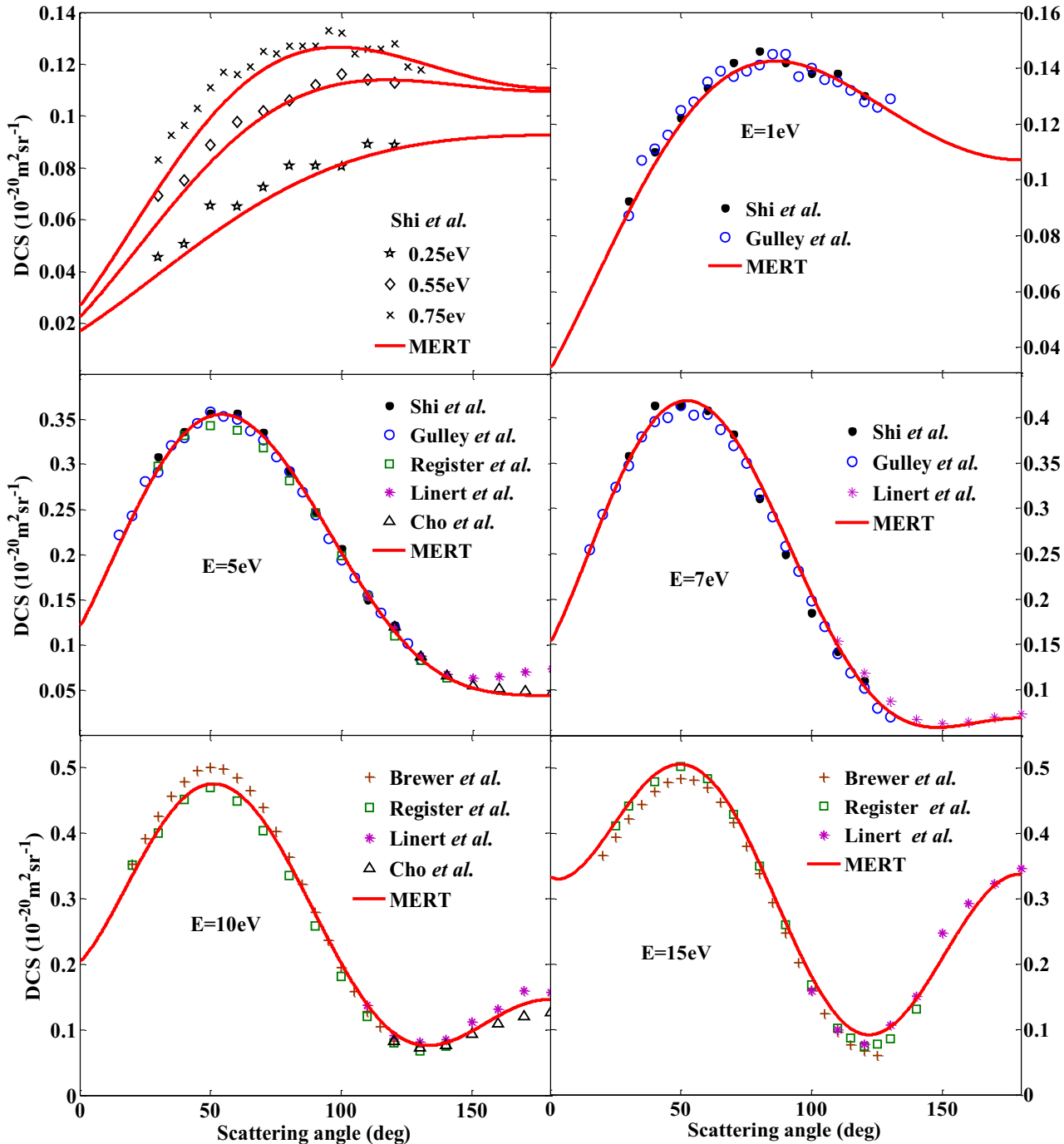

\section{Conclusions}

In this paper, the effective range analysis of electron interaction with noble gases, applied previously to helium, argon [34] and krypton [35], has been completed by studying $\mathrm{e}^{-}-\mathrm{Ne}$ collisions. It has been shown that non-relativistic modified effective range theory (MERT) in the form proposed by Idziaszek and Karwasz [36] can be used to describe elastic integral, momentum transfer and differential cross-sections for electron-neon collisions up to $16 \mathrm{eV}$, i.e. the threshold for the first inelastic process. The effectiveness of the model confirms that elastic collisions of slow electrons with neon (and other noble gases) are dominated by the long-range adiabatic polarization potential ( $\left.V_{\text {pol }}=-\alpha / 2 r^{4}\right)$ while the contribution of the shortrange potential (related with a complex multi-body interaction) to the scattering phase shifts of $s-, p-$ and $d-$ partial waves is very well approximated by simple quadratic expressions - the effective range expansions. It was shown that only six effective range parameters are sufficient to characterize energy and angular variations of whole elastic scattering cross-sections. This is an improvement when compared to the equivalent but fully empirical sevenparameter model proposed by O'Malley and Crompton [31]. Moreover, in contrast to the latter approach, the present model describes large sets of elastic cross-sections using only one equation for the scattering phase shifts experienced by dominating partial waves.

The comparison of empirically determined effective range parameters for neon with those found for other noble gases [34, 35] does not reveal any particular systematics. Nevertheless, more theoretical efforts are needed in order to understand the physical interpretation of these parameters and to relate the effective range approximation with the real interaction potential. This could be a useful subject for the inverse scattering theory aiming to recover unknown short-range potential from a set of scattering data. Moreover, the relativistic version of MERT [52] can possibly 
extend the applicability of the present theory to much heavier targets such as xenon (Xe) where subtle spin-orbit interactions play an important role. This work is in progress.

Acknowledgments The author thanks Prof. G. Karwasz and Dr. hab. Z. Idziaszek for the useful remarks. The financial support of the Foundation for Polish Science and the Polish Ministry of Science and Higher Education is acknowledged.

Open Access This article is distributed under the terms of the Creative Commons Attribution License which permits any use, distribution, and reproduction in any medium, provided the original author(s) and the source are credited.

\section{References}

1. X. Shi, P.D. Burrow, J. Phys. B: At. Mol. Phys. 25, 4273 (1992)

2. L.L. Alves, K. Bartschat, S.F. Biagi, M.C. Bordage, L.C. Pitchford, C.M. Ferreira, G.J.M. Hagelaar, W.L. Morgan, S. Pancheshnyi, A.V. Phelps, V. Puech, O. Zatsarinny, J. Phys. D: Appl. Phys. 46, $334002(2013)$

3. K. Shigemura, M. Kitajima, M. Kurokawa, K. Toyoshima, T. Odagiri, A. Suga, H. Kato, M. Hoshino, H. Tanaka, K. Ito, Phys. Rev. A 89, 022709 (2014)

4. I. Linert, B. Mielewska, G.C. King, M. Zubek, Phys. Rev. A 74, 042701 (2006)

5. H. Cho, R.P. McEachran, S.J. Buckman, H. Tanaka, Phys. Rev. A 78, 034702 (2008)

6. A. Salop, H.H. Nakano, Phys. Rev. A 2, 127 (1970)

7. T.S. Stein, W.E. Kauppila, V. Pol, J.H. Smart, G. Jesion, Phys. Rev. A 17, 1600 (1978)

8. J. Ferch, W. Raith, unpublished cross section data from the Bielefeld group, 1985. Numerical data taken from supplemental material of ref. [16]

9. J.C. Nickel, K. Imre, D.F. Register, L. Vuskovic, S. Trajmar, J. Phys. B: At. Mol. Phys. 18, 125 (1985)

10. V. Kumar, E. Krishnakumar, K.P. Subramanian, J. Phys. B: At. Mol. Phys. 20, 2899 (1987)

11. R.J. Gulley, D.T. Alle, M.J. Brennan, M.J. Brunger, S.J. Buckman, J. Phys. B: At. Mol. Phys. 27, 2593 (1994)

12. C. Szmytkowski, K. Maciag, G. Karwasz, Phys. Scr. 54, 271 (1996)

13. W.Y. Baek, B. Grosswendt, J. Phys. B: At. Mol. Phys. 36, 731 (2003)

14. D.F. Register, S. Trajmar, Phys. Rev. A. 29, 1785 (1984)

15. D.F.C. Brewer, W.R. Newell, S.F.W. Harpers, A.C.H. Smith, J. Phys. B: At. Mol. Phys. 14, L749 (1981)

16. Y. Cheng, L.Y. Tang, J. Mitroy, M.S. Safronova, Phys. Rev. A 89, 012701 (2014)

17. O. Zatsarinny, K. Bartschat, Phys. Rev A 85, 062710 (2012)

18. O. Zatsarinny, K. Bartschat, Electron Scattering Database (2012). http://www.lxcat.laplace.univ-tlse.fr
19. H.P. Saha, Phys. Rev. A 39, 5048 (1989)

20. H.P. Saha, Phys. Rev. Lett. 65, 2003 (1990)

21. A.W. Yau, R.P. McEachran, A.D. Stauffer, J. Phys. B: Atom. Mol. Phys. 11, 2907 (1978)

22. R.P. McEachran, A.D. Stauffer, J. Phys. B: At. Mol. Phys. 16, $4023(1983)$

23. R.P. McEachran, A.D. Stauffer, Phys. Lett. A 107, 397 (1985)

24. A. Dasgupta, A.K. Bhatia, Phys. Rev. A 30, 1241 (1984)

25. E.A. Garbaty, R.W. LaBahn, Phys. Rev. A 4, 1425 (1971)

26. D.G. Thompson, J. Phys. B: Atom. Mol. Phys. 4, 468 (1971)

27. W.C. Fon, K.A. Berrington, J. Phys. B: At. Mol. Phys. 14, 323 (1981)

28. C. Bottcher, J. Phys. B: At. Mol. Phys. 4, 1140 (1971)

29. M.R.C. McDowell, J. Phys. B 4, 1649 (1971)

30. J.F. Williams, J. Phys. B: Atom. Mol. Phys. 12, 265 (1979)

31. T.F. O'Malley, R.W. Crompton, J. Phys. B: Atom. Mol. Phys. 13, $3451(1980)$

32. T.F. O’Malley, L. Spruch, L. Rosenberg, J. Math. Phys. 2, 491 (1961)

33. T.F. O'Malley, L. Rosenberg, L. Spruch, Phys. Rev. 125, 1300 (1962)

34. K. Fedus, G.P. Karwasz, Z. Idziaszek, Phys. Rev. A 88, 012704 (2013)

35. K. Fedus, Phys. Scripta 89, 105401 (2014)

36. Z. Idziaszek, G. Karwasz, Phys. Rev. A 73, 064701 (2006)

37. Z. Idziaszek, G. Karwasz, Eur. Phys. J. D 51, 347 (2009)

38. S.J. Buckman, J. Mitroy, J. Phys. B: At. Mol. Phys. 22, 1365 (1989)

39. K. Fedus, G. Karwasz, Eur. Phys. J. D 68, 93 (2014)

40. T.N. Olney, N.M. Cann, G. Cooper, C.E. vBrion, Chem. Phys. 223, 59 (1997)

41. M.K. Ali, P.A. Fraser, J. Phys. B: At. Mol. Phys. 10, 3091 (1977)

42. V. Godyak, R. Piejak, Phys. Rev. Lett. 65(8), 996 (1990)

43. Z. Navratil, P. Dvorak, O. Brzobohaty, D. Trunec, J. Phys. D: Appl. Phys. 43, 50 (2010)

44. R. Szmytkowski, Phys. Rev. A 51, 853 (1995)

45. S.J. Buckman, B. Lohmann, J. Phys. B: Atom. Mol. Phys. 19, 2547 (1986)

46. A.G. Robertson, J. Phys. B: Atom. Mol. Phys. 5, 648 (1972)

47. T. Koizumi, H. Murakoshi, S. Yamamoto, I. Ogawa, J. Phys. B: Atom. Mol. Phys. 17, 4387 (1984)

48. Biagi v7.1 and v8.9 - Cross sections extracted from program MagBoltz, version 7.1 and 8.9 respectively, March 2010, in Electron Scattering Database, http://www.lxcat.laplace.univ-tlse.fr

49. W. Lowell Morgan, Cross sections extracted from Kinema Research Software, in Electron Scattering Database, http://www. lxcat.laplace.univ-tlse.fr

50. V. Puech, LPGP, Orsay, France: Cross section sets assembled by V Puech and colleagues in late 1980's and 1990's, in Electron Scattering Database, http://www.lxcat.laplace.univ-tlse.fr

51. The SIGLO database: it is the "in-house" data used by the group GREPHE at LAPLACE in Toulouse, in Electron Scattering Database, http://www.lxcat.laplace.univ-tlse.fr

52. C. Bahrim, U. Thumm, I.I. Fabrikant, J. Phys. B: At. Mol. Opt. Phys. 34, L195 (2001) 